\title{
Why Geometry Radiates: Quantum Gravity as Perspective
}

\author{
Rob Langley \\ Norwich, UK \\ Email: rhlangley@hotmail.co.uk
}

Received 20 January 2016; accepted 22 March 2016; published 25 March 2016

Copyright (C) 2016 by author and Scientific Research Publishing Inc.

This work is licensed under the Creative Commons Attribution International License (CC BY). http://creativecommons.org/licenses/by/4.0/

(c) (i) Open Access

\begin{abstract}
It is reasonably expected 1) that a theory of quantum gravity will unify the extremes of scale currently described by General Relativity and quantum mechanics, and 2) that black holes are the crucible from which a theory of quantum gravity will emerge. In perspective, we already have a mechanism that links the local, macroscopic frame with the remote, apparently microscopic frame. A simple mathematical principle acts as a limit on $\mathrm{D}(n)$, suggesting a "maximum physical reality", and that effects which are clearly perspectival at $\mathrm{D}=3$ become "more real" (effectively observerindependent) with each $D(n)$ increment. The model suggests alternative interpretations of gravitation and the quantum, entanglement, space, the Standard Model of particles and interactions, black holes, the measurement problem and the information paradox.
\end{abstract}

\section{Keywords}

Quantum Gravity, Black Holes, Entanglement, Firewalls, ER = EPR , Information Paradox

\section{Introduction}

Profound conceptual incompatibilities exist between General Relativity (GR) and quantum mechanics (QM) as currently formulated. For instance; 1) whereas in GR, time is just another covariantly transforming coordinate of an arbitrarily differentiable spacetime manifold, in QM it is an external, Newtonian parameter and properties are discrete, and 2) the respective macroscopic and microscopic domains of applicability remain as disconnected as ever. The paper proposes perspective at $\mathrm{D}>3$ as a possible means of unification. A simple mathematical principle is then invoked as a natural limit on $\mathrm{D}(n)$ and the implied existence of a "maximum physical reality" therefore means that effects that are clearly perspectival at $\mathrm{D}=3$ must become "more real" (effectively observer-independent) with each $\mathrm{D}(n)$ increment. (The apparently microscopic thus becomes the effectively mi- 
croscopic, and 3-space scale extremes are juxtaposed.) Characteristics of the relationship between the earthbound observer and the horizon are applied to $\mathrm{D}>3$, suggesting perspectival interpretations of space, universal expansion, radiation, entanglement, gravitation, mass, dimensional reduction and measurement (as projection rather than amplification). The difference between apparent flatness of the 2-surface and actual global closure corresponds to the boson of the gauge interpretation (i.e., the observer's height, projected from the local, macroscopic frame to the remote, effectively microscopic frame, where it appears as a "collapse vector"). Although horizons therefore appear to radiate, a Hawking photon is instead thought of as a "unit of projection" (of the local frame to the remote), thus challenging the notion that photons "travel through space".

The paper is structured as follows. Section 2 considers the natural limit on $\mathrm{D}(n)$ and its implications. The main geometrical characteristics of $\mathrm{D}(3 \leq n \leq 5)$ are outlined in Section 3, including a duality of the fundamental constants of GR and QM (i.e., $v=c$ and $E=h v$ ). Section 4 interprets perspective as gauge, and Section 5 then interprets the Standard Model gauge group as perspective, with gravity included by way of chargemass duality. Characteristics from Section 3 are then applied to black holes in Section 6 using an Alice-and-Bob scenario. A scheme for quantum gravity (QG) is outlined, together with an account of black hole entropy and an interpretation of the information paradox as a perspectival effect. Finally, Section 7 is a discussion and brief summary of the main ideas. In terms of notation, the case in which a $\mathrm{D}(n)$ surface is spherically closed and embedded in $\mathbb{R}^{\mathrm{D}(n+1)}$ is denoted typically as $\mathrm{D}(n) \mid \mathrm{D}(n+1)$.

\section{Natural Limit on $\mathrm{D}(n)$}

References $s_{1}$ and $s_{2}$ are given (Figure 1), and at each iteration, a mid-point or difference is specified between the references, using the previously-established difference as the new reference. (After the second iteration, the line of references approaches the difference $s_{1}-s_{2}$ asymptotically.) After three iterations (the dashed line) the difference is completely detached from the original references.

If differences are now expressed as derivatives, then the difference between two spacetime positions $s_{1}$ and $s_{2}$ is a change of position or velocity $(\mathrm{d} s / \mathrm{d} t)$, and the difference between two velocities is an acceleration $\left(\mathrm{d}^{2} s / \mathrm{d} t^{2}\right)$, etc. At each iteration, the previously established difference is used as a new reference, and the detaching again occurs between iterations 3 and 4 . Thus, at iteration $n=3$ the original references are "superseded" (the original reference $s_{1}$ only appears inside brackets after this level). Assuming the applicability of this simple principle, the obvious claims would be 1) that the Universe in effect "runs out of references" by which to distinguish differences (i.e., that the Universe only differentiates twice with respect to time), 2) that $\mathrm{D}(n)$ is effectively indistinguishable beyond D6 and 3) that physical observables are therefore also limited. This also implies that there is a "maximum physical reality" at D6, that effects that are clearly perspectival at D3 become "more real" (effectively observer-independent) with each $\mathrm{D}(n)$ increment, that $\mathrm{D} 7$ is nonphysical (i.e., mathematical) and that D8 may be associated with observer objectivity.

\section{Diminishing Freedoms}

Since the observer also possesses limited freedoms, perspectival effects at $\mathrm{D}=3$ (e.g., geometry, horizons) become increasingly observer-independent with each $\mathrm{D}(n)$ increment with respect to the observer; i.e., the

\begin{tabular}{|c|c|c|c|c|}
\hline & $S_{1}$ & & $S_{2}$ & \\
\hline 0 & $\mathbf{I}$ & & $\mathbf{I}$ & $s_{1}, s_{2}$ \\
\hline 1 & ] & $\mathbf{I}$ & {[} & $s_{1}-s_{2}$ \\
\hline 2 & ] & $\mathbf{I}$ & {[} & $s_{1}-\left(s_{1}-s_{2}\right)$ \\
\hline 3 & ] & I $\quad$ ] & ] & $\left(s_{1}-\left(s_{1}-s_{2}\right)\right)-\left(s_{1}-s_{2}\right)$ \\
\hline 4 & ] & {$\left[\begin{array}{ll}2 & -1\end{array}\right.$} & ] & $\left(\left(s_{1}-\left(s_{1}-s_{2}\right)\right)-\left(s_{1}-s_{2}\right)\right)-\left(s_{1}-s_{2}\right)$ \\
\hline 5 & ] & {$\left[\begin{array}{lll}7 & 0 & {[\mathbf{I}}\end{array}\right]$} & [] & $\left(\left(\left(s_{1}-\left(s_{1}-s_{2}\right)\right)-\left(s_{1}-s_{2}\right)\right)-\left(s_{1}-s_{2}\right)\right)-\left(s_{1}-s_{2}\right)$ \\
\hline
\end{tabular}

Figure 1. Successive iterations of a simple difference operation reach a critical threshold at $n=3$. 
observer possesses diminishing freedoms with respect to physical phenomena. Therefore we define dimensionality in terms of freedoms. Whereas in $\mathbb{R}^{3}$ the observer possesses the freedom to orientate in any superposition of axes $\{x, y, z\}$, in $\mathbb{R}^{3,1}$ the observer has limited freedom to orientate in 4-space in axes $\{x, y, z, t\}^{1}$, and in $\mathbb{R}^{5}$, freedom is further curtailed; a body now has mass (i.e., is subject to spacetime curvature). Similarly for horizons; at D2|D3 the $S^{1}$ planetary horizon is global (remote), surrounds the observer, and is completely observer-dependent, moving as the observer changes 2-position (i.e., is totally "permeable"). At D3|D4 the $S^{2}$ cosmological horizon is also global (remote) and surrounds the observer, but is less observer-dependent, moving as the observer changes 3-position (i.e., with the observer's velocity) but being causally "semi-permeable". Finally, at D4|D5, massive bodies are now subject to local (multiple) $S^{3}$ black hole horizons which do not surround the observer (rather, it is as though a remote, global $S^{3}$ horizon has been brought into the local frame, in some sense). The event horizon is now a strictly one-way membrane with unclear observer-dependent status; one would expect it to accelerate with the observer. (The Rindler horizon, with similar thermal properties, appears at constant 3-distance for constant acceleration.)

\section{Geometrical Characteristics}

Perspective in 3-space is that effect whereby a receding object appears to shrink to an apparent point. Perspective plus curvature (e.g., on the closed surface of Earth) means that the size of the object does not go to zero before the object disappears over the horizon. At D2|D3, using metres as units, Alice stands with 3-height $h_{3}=2$ above the 2-surface, which is closed with constant radius of curvature $r_{2}=6.371 \times 10^{6}$. More formally, we specify a manifold $\mathcal{M}^{2}=S^{2}$ that is closed with constant radius of curvature $\left(r_{2}\right)$ and embedded in $\mathbb{R}^{3}$ according to the Nash theorems. At $x \in \mathcal{M}^{2}$ we define a 3-height $\left(h_{3}\right)$ and a tangent space $T_{x} \mathcal{M}^{2}$ bounded at a constant distance $\ell$ from $x$ by $S_{(A)}^{1}$, where $A \in T_{x} \mathcal{M}^{2}$. The manifold $\mathcal{M}^{2}$ is bounded by $S_{(B)}^{1}$, where $B \in \mathcal{M}^{2}$ at a distance along $\mathcal{M}^{2}$ such that $\ell\left(S_{(A)}^{1}-S_{(B)}^{1}\right)=h_{3}$, where $T_{B} \mathcal{M}^{2}$ is coincident with the "top" of $h_{3}$. The tangent space $T_{x} \mathcal{M}^{2}$ is defined as the perception of $\mathcal{M}^{2}$ due to $h_{3} \lll r_{2}$ in the frame of an observer located at $x$ with $h_{3}$. We note the following characteristics.

1) Assuming $h_{3} \lll r_{2}$, the 2-surface looks flat to good approximation (Figure 2(a)).

2) There is an increasing divergence between apparent flatness and actual global closure (Figure 2(b)).

3) Parallel lines on the surface (e.g., railway lines) appear to converge to an apparent D0 point or singularity on the $S^{1}$ horizon (at a distance $\ell \approx 5.05 \times 10^{3}$ ).

4) With increasing distance from Alice, objects appear to contract and space appears to expand, and Alice reasons that these rates must be exactly equivalent; $\mathbf{C O N} \equiv \mathbf{E X P}$.

5) There is a $\mathrm{D}(n-3)$ dimensional reduction from Alice's 3-height to an apparent D0 point on $S^{1}$.

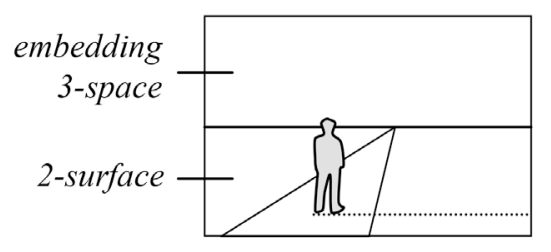

(a)

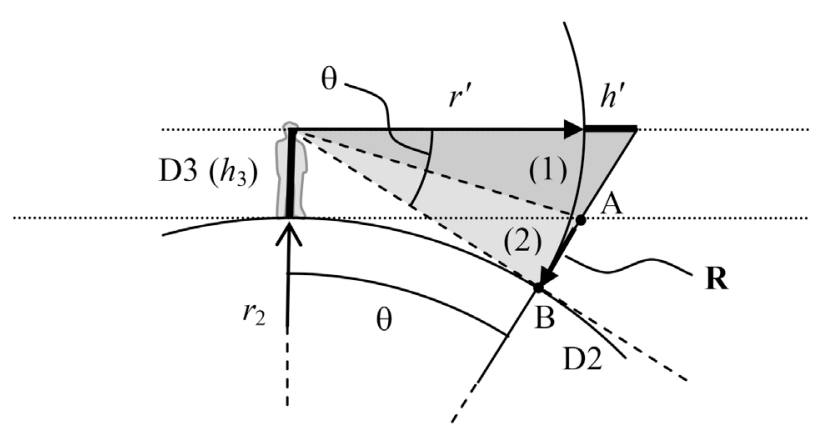

(b)

Figure 2. The familiar geometry of the earthbound D2|D3 scenario.

${ }^{1}$ If time is treated as an imaginary coordinate $(\sqrt{-1} c t)$, the Lorentz transformation $\gamma(v)=1 / \sqrt{\left(1-\left(v^{2} / c^{2}\right)\right)}$ can be thought of as a rotation in Euclidean 4-space, $\mathbb{R}^{4}$. 
6) There are two such points; A, where Alice (naively) believes she is looking, and $B$, where she is actually looking. In 3-space, these are clearly the same point; $A$ and $B$ are identified $(A \equiv B)$.

7) If Alice gains sufficient 3-height, she will see the D0 point singularity transform or open up to become a D1 line singularity (i.e., there is a non-zero separation, $\ell>0$, between railway lines as they disappear over the horizon). This forced transition, $\mathrm{A} \rightarrow \mathrm{B}$, acts as a gate that reveals the embedding of $\mathcal{M}^{2}$ in $\mathbb{R}^{3}$ (i.e., the D2|D3 case).

8) The difference between apparent flatness and actual global closure is just Alice's local 3-height $\left(h_{3}\right)$, projected (and rotated through $\theta=0.045^{\circ}$ ) to the remote, $S^{1}$ horizon. (Alice's apparent 3-height, as projected to the remote frame and seen from the local frame, is less than the thickness of the average human hair; $h_{3(L O C)} \rightarrow\left(h_{3(R E M)} \approx 6.3 \times 10^{-5}\right)$.)

9) The angle $\theta=0.045^{\circ}$ subtended at Alice's eye between the true horizontal line of sight (i.e., tangential at a radius of $r=r_{2}+h_{3}$ ) and a line of sight to the actual horizon is necessarily equal to that subtended at the centre of the Earth between radial lines to the locations of Alice and the horizon, so the relationship (and transition) between points A and B is proportional to and associated with those between the local observer and the remote horizon.

10)The transition $\mathbf{R}=(\mathrm{A} \rightarrow \mathrm{B})$ is also that from triangle (1) with $a^{2}=b^{2}+c^{2}+2 \cos \theta$, to triangle (2), with $a^{2}=b^{2}+c^{2}$, so the transition $\mathrm{A} \rightarrow \mathrm{B}$ is associated with the loss of the $2 \cos \theta$ term.

11)Observer-dependency of the $S^{1}$ horizon can be expressed as the fact that 2-distance to the $S^{1}$ horizon is a constant in every frame.

12)The $h_{3}$ of an object that disappears over $S^{1}$ does not cease to exist but is "conserved" in 3-space.

At D3 $\mid \mathbf{D} 4$, Alice's 4-height is taken to be velocity (rather than the Minkowski spacetime interval, $\left.\Delta s^{2}=\Delta x^{2}+\Delta y^{2}+\Delta z^{2}-(c \Delta t)^{2}\right)$. Assuming $h_{4} \lll r_{3}$, the 3-surface "looks flat”, but due to the existence of $S^{2}$, the apparent velocity of Bob, receding, "contracts" with 3-distance to some irreducible value, interpreted here as a minimum energy, $\hbar=1.055 \times 10^{-34} \mathrm{~m}^{2} \mathrm{~kg} / \mathrm{s}$. However, when Bob is at $S^{2}$, his velocity relative to Alice is $v=c$, and so the curvature limits are the characteristic GR and QM constants, $v=c$ and $E=h v$. At D3|D4

the linear expansion of spacetime with increasing 3-distance from Alice corresponds to universal expansion with constant velocity [1] [2]. We also note that observer-dependency of the $\boldsymbol{S}^{2}$ (cosmological) horizon can be expressed in terms of the fact that 3-distance to the $S^{2}$ horizon is a constant in every frame.

At D4|D5, Alice's 5-height is acceleration. Assuming $h_{5} \lll r_{4}$, the 4-surface "feels flat"; i.e., implying a physical basis for $m_{i} \equiv m_{g}$, as required by the Equivalence Principle (EP), but due to the existence of $S^{3}$, the apparent acceleration of Bob, falling towards the black hole event horizon, decreases with 4-distance to some irreducible value, interpreted here as a minimum acceleration, $\lambda=1 \times 10^{-52} \mathrm{~m}^{-2}$. At the same time, Bob's acceleration relative to Alice is $a=\varphi$, where $\varphi$ is an effective maximum beyond which the Unruh or Hawking radiation wavelength is $\omega_{\varphi}<\ell_{P}$ (i.e., a firewall) and so the curvature limits are $\varphi$ and $\lambda$. At D4|D5, the second-order expansion of spacetime with increasing 3-distance corresponds to accelerating universal expansion [3] [4]. We also note that observer-dependency of the $\boldsymbol{S}^{\boldsymbol{3}}$ (black hole) horizon can be expressed as the fact that 4-distance $(v=c)$ to the $S^{3}$ horizon is a constant in every frame. Thus the fixed 4-distance $(v=c)$ to the $S^{3}$ black hole event horizon in the local, macroscopic frame is the quantum of energy $(E=h v)$ in the remote, effectively microscopic frame. In this sense, then, RELATIVITY $\equiv$ QUANTISATION $^{2}$.

\section{Perspective as Gauge}

Alice and Bob are astronauts moving away from each other in empty space, and, for the purposes of this consideration, we assume that neither has any prior spatial understanding. Alice first notes that Bob appears to be shrinking. Since they disagree on who is shrinking, they each theorise the existence of an embedding 3-space $\mathbb{R}^{3}$ that makes sense of the fact that, with increasing distance, objects appear to contract (CON) and space appears to expand (EXP). Now as observers on Earth (but still without any geometrical understanding) Alice and

\footnotetext{
${ }^{2}$ The author is not aware that a direct equivalence has been proposed in previous works by other authors.
} 
Bob stand with equal 3-height $\left(h_{3}\right)$ above the 2-surface, which is closed with constant radius of curvature $\left(r_{2}\right)$. Alice is directly aware of the existence of the 2-space, but has to infer (e.g., using parallax) the existence of a higher, 3-space. Assuming $h_{3} \lll r_{2}$, the 2-surface looks flat, so Alice does not recognise $S^{1}$ as an horizon, but merely as a "dimensional separation". Therefore the significance of 3-height (or potential) and its relation to the horizon remains unacknowledged. In other words, Alice does not recognise Earth as an object.

However, from outside her frame, we can see that the difference in her frame between apparent flatness and actual global closure is just the potential $\left(h_{3}\right)$, projected (and rotated through $\theta$ ) to the remote horizon, represented by the "collapse" vector $\mathbf{R}$ in Figure 2(b). Now Alice and Bob disagree about shrinkage and phase, which vary from point to point. In gauge terms, the 2-surface is the base space $\mathcal{M}$, and the unacknowledged potential is the fibre $\mathcal{V}$, defining an internal space at each point on $\mathcal{M}$ which together comprise a fibre bundle, $\mathcal{B}$, such that $\mathcal{B}=\mathcal{M} \times \mathcal{V}$. The gauge field comprises gauge connections that correspond to the group $\mathcal{G}$ of continuous symmetries of $\mathcal{B}$, and in that there is a canonical projection, submersion or surjective map, $\pi: \mathcal{B} \rightarrow \mathcal{M}$, then the phase $(\theta)$ is a continuous image of $\mathcal{M}$ in $\mathcal{B}$. Thus, $\mathbf{R}$ is just the potential $\left(h_{3}\right)$, projected from the local to the remote frame, that appears in Alice's frame as a "minimum unit" (i.e., the gauge boson or field quantum) that is the coupling constant or interaction strength that translates between frames and restores local gauge invariance. Alice also notes that as she and Bob stand at each other's horizon, the values 2 and $6.3 \mathrm{~m} \times 10^{-5}$ define curvature limits at D2 $\mid \mathrm{D} 3$, allowing this geometry to be applied to $\mathrm{D}>3$.

\section{Gauge as Perspective}

The Standard Model (SM) is the non-Abelian gauge group $\mathrm{SU}(3) \times \mathrm{SU}(2) \times \mathrm{U}(1) / \mathrm{Z}_{6}$ that describes particles and interactions in terms of a sequence of early-epoch phase transitions following a Big Bang at $t=0$. In the perspective approach, the SM comprises two groups of sets of $\mathrm{D}(n>3)$ perspective reductions. The first is $\{\mathrm{D}(4 \leq n \leq 8)\} \rightarrow\{\mathrm{D}(1 \leq n \leq 5)\}$, where $\mathrm{D}(n>3)$ are derivatives, $\left(\mathrm{d}^{n} \mathrm{~s} / \mathrm{d} t^{n}\right)$. These generate apparent Planck dimension perspectival convergence limits $\ell_{P}^{n}$ (with $n_{D}=n_{P}$ ), which are interpreted as fermions. At each $\mathrm{D}(n)$ level, a $\mathrm{D}(n+1)$ height is associated with spherical closure of $\mathrm{D}(n)$, forming a series of "onion layers", where each closure $f(C): D(n)$ generates $\mathrm{D}(n)$-forms that populate $\mathrm{D}(n)$ space; $f(C): D(n=0) \rightarrow D(n=1)$ (i.e., a string), etc, as depicted in Figure 3. The second group is $\{\mathrm{D}(n=4,5)\} \rightarrow\{\mathrm{D}(n=1,2)\}$, generating apparent $\mathrm{D} 2$ area $\left(\ell_{P}^{2}\right)$ singularities that live on the $S^{3}$ horizon separating D4|D5, interpreted as the leptons, the 2-forms that in the SM are "cousins" to quarks.

Assuming $h_{\mathrm{D}(n+1)} \lll r_{\mathrm{D}(n)}$ at each level, each $\mathrm{D}(n)$ surface can be described as a state defined by $n$ directions or generators, so each lower level can be thought of as a state-splitting or copying process producing families of $\mathrm{D}(n-x)$ generations; e.g., the Cabibbo-Kobayashi-Maskawa matrix describing expectation-values for transitions between families and generations of quarks (with the Cabibbo angle corresponding to probabilities of decay modes), and the Pontecorvo-Maki-Nakagawa-Sakata matrix summarising weightings for lepton-mixing. The superpositions of directions (generators) at higher $\mathrm{D}(n)$ are rotations that transform lower $\mathrm{D}(n)$ particles, as depicted in Figure 4.

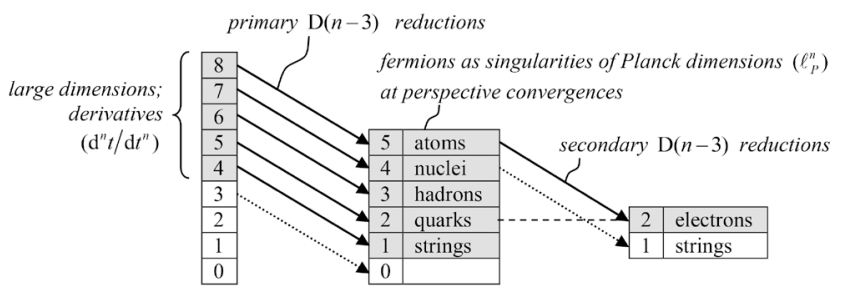

Figure 3. Two groups of $\mathrm{D}(n-3)$ reductions generate an "onion-layer" model of particles and interactions, and juxtapose quarks and leptons (dashed line). 
D4 (4)

(4)

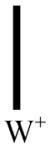

D3

(12)

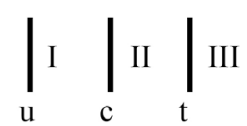

D2

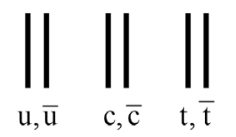

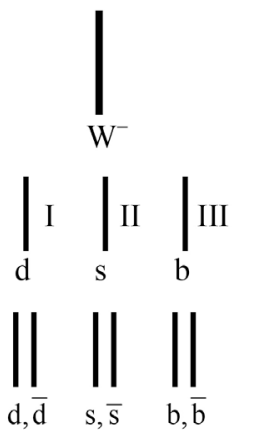

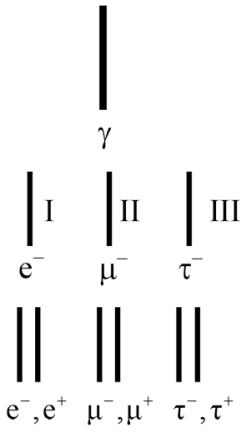

Figure 4. $\mathrm{D}(n)$ directions are particles at $\mathrm{D}>n$, and IVBs at $\mathrm{D}>n$ are fermionic generations at $\mathrm{D}<n$. In this progression, space at $\mathrm{D}(n)$ becomes a fermion at $\mathrm{D}(n+1)$.

Each inter-dimension or phase transition is a $\mathrm{D}(n-3)$ perspective reduction; at each level, a $\mathrm{D}(n)$-form possesses a local $\mathrm{D}(n+1)$ height (a property defined by that internal space), from which there is an apparent diminishing of $h_{\mathrm{D}(n+1)}$ until it disappears over the associated $S^{\mathrm{D}(n-1)}$ horizon. Thus, SU(3) colour bleaches out of Quark-world as quarks "fall into Quark-world black holes” (i.e., inside-out hadrons), which is the process of baryogenesis, marking the global closure of D2, the formation of colour-neutral baryons and the conservation of colour. Likewise, charge bleaches out of Nucleus-world as "nuclei fall into Nucleus-world black holes" (i.e., electrons, or inside-out atoms), corresponding to the formation of charge-neutral atoms and the conservation of charge at global closure of D4. In the perspective model at D4|D5, information bleaches out of the (D4) universe as an SM phase transition. In QED, the atomic nucleus containing positively charged protons is surrounded and perfectly balanced by shells populated by negatively charged electrons; $N_{P} \equiv N_{e^{-}}$. The 5-height of the nucleus connects via $\mathrm{D}(n-3)$ perspective reduction to a Dirac spinor of the Penrose zigzag electron, $\psi=\left(\alpha_{A}, \beta_{A^{\prime}}\right)$ [5] in the last electron shell. In the nucleus frame, assuming $h_{5} \lll r_{4}$, the separation of equally spaced calibrations of the $n$-surface appears to decrease with increasing distance and so the exact location of the $S^{3}$ horizon is uncertain, and electron shells are thus interpreted as multiple $S^{3}$ horizons. The quantum state of the atom is defined as a superposition of all shells, or $|\psi\rangle_{\text {Атом }}=c_{1} \psi_{1}+c_{2} \psi_{2}+c_{3} \psi_{3}+\cdots+c_{n} \psi_{n}$, in which $\psi_{1}, \psi_{2}, \psi_{3}, \cdots, \psi_{n}$ are the eigenstates of an observable (e.g., the principle quantum number, $n$ ) corresponding to eigenvalues $a_{1}, a_{2}, a_{3}, \cdots, a_{n}$.

\section{Electromagnetism-Gravitation Duality}

Given the correspondence between $v=c$ at the macroscopic level and $E=h v$ at the microscopic level, we now ask if there is a precedent for the notion of charge at the macroscopic scale. We note that mass tends towards horizons; either expanding (state; EXP) as part of the Hubble flow towards the $S^{2}$ cosmological horizon, or contracting (state; CON) towards $S^{3}$ black hole event horizons (Figure 5). In Alice's $h_{5}$ frame, the rates must be exactly equal; $(\mathrm{d} / \mathrm{d} t) \mathbf{E X P} \equiv(\mathrm{d} / \mathrm{d} t) \mathbf{C O N}$. Such labelling is arbitrary and therefore cannot be defined in any absolute way. In gauge-theoretic terms, just as the photon expresses the fact that we cannot define charge in an absolute sense in the Coulomb gauge, so the graviton would be the gauge particle mediating between EXP and CON "charges" .

Generalising Newton's third law, any derivative of action-force $\overrightarrow{\mathbf{F}}_{A C T \text {. }}=\mathrm{d}^{n} \mathrm{~s} / \mathrm{d} t^{n}$ applied to a massive body (regardless of mass or composition) will elicit an instantaneous, exactly equivalent and perfectly counter-directed reaction-force, $\overrightarrow{\mathbf{F}}_{\text {REACT. }}=\mathrm{d}^{n} \mathrm{~s} / \mathrm{d} t^{n}$. Therefore, the picture suggested is of a massive 3-object (a CON

\footnotetext{
${ }^{3}$ It is noteworthy that in Penrose's Conformal Cyclic Cosmology model [6] [7], conformal invariance prevails both near an initial singularity and at a future state of maximum spatial expansion (future boundary hypersurface $\mathcal{I}$ ), thus the state of maximum expansion is converted to the state of maximum contraction.
} 


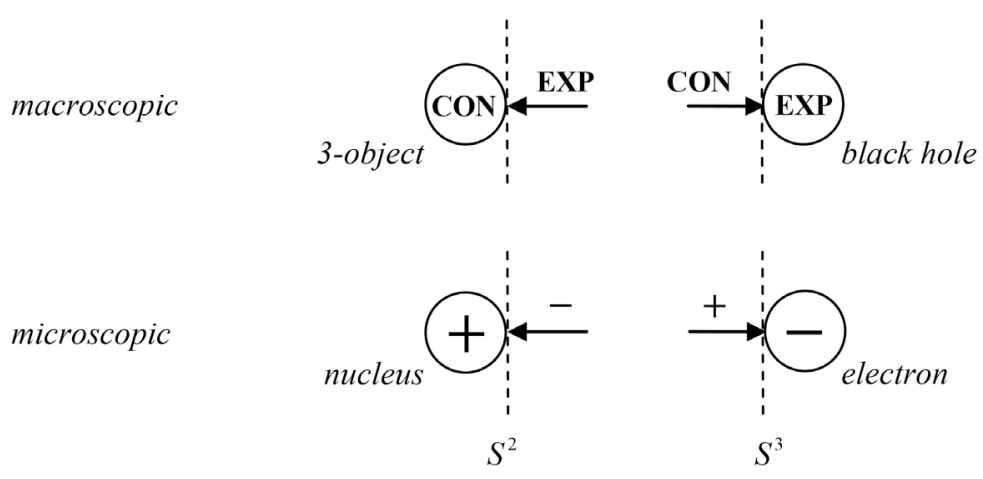

Figure 5. At the microscopic level, mass tends either towards the $S^{2}$ (cosmological) horizon as part of the Hubble flow (EXP) or towards the $S^{3}$ (black hole event) horizon $(\mathrm{CON})$, just as at the microscopic level, charged particles tend either towards the nucleus $(+)$ or the electron $(-)$.

charge) surrounded by "derivative shells" populated by black holes. The $m_{g}$ of the 3-object is perfectly balanced by the $m_{i}$ of the derivative shells, and so the QG definition of freefall would be the superposition of all derivative shells. Therefore mass at the macroscopic level is charge at the microscopic level. In the perspective model, projection of height in the local, macroscopic frame to the remote (effectively) microscopic frame becomes more real, until, at the level of maximum physical reality, it is effectively real. That means that the observer is effectively projected into the microscopic domain, implying a thorough mixing of macroscopic and microscopic scales, and, somewhat outrageously, that electrons are black holes.

Black hole-electron duality (as suggested in [8]-[10]) opens up many further correspondences. For instance, QED vacuum polarisation, in which the bare electron charge is dressed by slight displacement of virtual $e^{-}+e^{+}$pairs away from and towards the charge, respectively, would correspond to GR vacuum polarisation, in which the bare black hole "EXP" charge is dressed by displacement of virtual charges, dark energy (EXP) and dark matter (CON), respectively. Also, QED renormalisation, in which the cancellation of divergent intergrals may be achieved by removing the electron interior region $r<r_{e}$ (where $r_{e}$ is the classical radius) would suggest that gravitational renormalisation would be likewise achieved by removal of the black hole interior region, $r<2 G M$.

We might also note that the perspective projection D5 $\rightarrow$ D2 maps the local, macroscopic (black hole) frame to the SM photon epoch following nucleosynthesis but before ions gained electrons. At this time, the densities of relativistic radiation (photons) and non-relativistic matter (atomic nuclei) were equal. The subsequent decoupling of matter and radiation and the formation of charge-neutral atoms corresponds in the perspective model to the global closure of D4. At recombination (the surface of last scattering), the photon mean path length became effectively infinite, and the Universe thus became transparent. At the macroscopic scale, the coincidence (or "why now?") problem asks why the energy densities of dark energy (i.e., the cosmological constant) and matter should be comparable. This suggests a perspective map $f(P):\left(\rho_{\lambda} \approx \rho_{M}\right) \rightarrow\left(\rho_{\gamma} \approx \rho_{M}\right)$, from the coincidence problem at the macroscopic scale to the pre-decoupling radiation-matter densities of the SM. In the perspective approach, gravitons at the macroscopic scale are photons at the microscopic scale, in which case, the experimental absence of gravitons would indicate that the Universe is not "yet" gravitationally transparent. We next consider black holes, the firewall question and the information paradox.

\section{Black Holes}

\subsection{Background}

If Hawking radiation is thermal [11], then the scattering matrix is nonunitary. Complementarity allows information to exist in the exterior and interior regions as long as observers cannot compare notes [12] [13], but the "AMPS" paper [14] showed inconsistencies in the theory's postulates, reasoning that if evaporation is unitary and maximal entanglement is monogamous, early radiation (C) and late radiation (B) must be entangled, giving 
rise to a firewall at $r=2 G M$ where entanglement between $\mathrm{B}$ and interior modes (A) is broken, or if the EP is to be preserved, then $B$ must be only entangled with $A$, at the expense of unitarity. The ER = EPR solution [15] is a duality between Einstein-Rosen bridges and EPR entanglement that allows entanglement of A and B (ensuring no drama), and entanglement of B and C via ER bridges (ensuring unitarity). Given certain computational complexity assumptions [16], Alice, by measuring Hawking radiation, generates a shockwave [17] in the ER bridge, thereby inducing a firewall in the remote black hole throat.

\subsection{Perspective Model Interpretation}

The effects listed in Section 2 may be demonstrated using Alice and Bob, in orbit around a massive black hole. They synchronise clocks and Alice remains in orbit while Bob begins his radial freefall descent towards the black hole. The effective divergence between their frames $\left(F_{A}\right.$ and $\left.F_{B}\right)$ is due to the fact that Bob is commoving with global accelerated expansion towards the local $S^{3}$, at which $a \rightarrow \max ^{4}$. Just as at D2|D3, apparent 3-height decreases with distance, so at D4|D5 in Alice's frame, Bob's apparent acceleration decreases as he approaches $r=2 G M$. The photons comprising his image redshift to $T_{\gamma}=T_{\mathrm{CMBR}}$, and Alice sees Bob hover (and therefore surmises that he must then burn up as a consequence of the EP). Calculating for the huge Lorentz and gravitational boosts, Alice records the exact time in her frame $t_{(r=2 G M)}\left(F_{A}\right)$ that Bob "actually" crosses $r=2 G M$. In Bob’s frame, the coordinate singularity $r=2 G M$ does not exist. Due to $h_{5} \lll r_{4}$, the 4-manifold feels flat, and so the apparent area singularity $\left(\ell_{P}^{2}\right)$ remains on the $S^{3}$ horizon at all times. (In Hamilton's analysis [19], the illusory horizon recedes in front of Bob until he finally merges with it in finite time at $r=0$.) The difference between apparent flatness (the tangent space, $T_{x} \mathcal{M}^{4}$ ) and actual global closure is just the observer's local 5-height (acceleration, or mass), projected to the remote horizon, where it appears as the gauge boson. Classically, one would say that geometry radiates, and assuming minimal 5-height (acceleration) due to $h_{5} \lll r_{4}$, then Hawking radiation (HR) is also minimal (i.e., HR $\propto \lambda$ ). However, in the perspective model, the more fundamental picture is projection of the local to the remote frame; the boson is the "unit of projection", so, strictly speaking, photons do not travel. Spatial extent is therefore emergent and the "3-ness" of space is just the fact of $\mathrm{D}(n-3)$ reduction; "space” is then thought of as the absence of intervening dimensionality.

At some point, Bob begins to experience tidal deformation. Now, according to the Correspondence Principle (CP), classical dynamics (typified by Newton's $\mathbf{F}=m a$ ) is recovered in the statistical limit and for well-defined wavepackets from quantum dynamics (typified by the Schrödinger equation, $i \hbar(\partial / \partial t)(|\psi\rangle \boldsymbol{r}, t)=\hat{H}(|\psi\rangle \boldsymbol{r}, t)$ ), and so the CP must therefore also relate the respective GR and QM “charges”, mass and information, and the associated dynamics (acceleration and measurement) by which each is registered. This validates a comparison between black holes and the slits in the Young double-slit experiment. Assuming no which-path detection, then in the frame of the emitted particle, the slits themselves must become superposed or wavelike. Therefore, if interference fringes are to appear, then just as the particle cannot "know" by which slit it is entering the set-up, so Bob cannot know towards which black hole he is falling. Bob is therefore falling into $N$ black holes and the tidal forces (fluctuations and oscillations) he experiences represent the superposition of distinct black hole spacetime geometries; $|\psi\rangle=\sum_{p=1}^{n} p=p_{1} g_{\mu \nu}^{(1)}+p_{2} g_{\mu \nu}^{(2)}+p_{3} g_{\mu \nu}^{(3)}+\cdots+p_{n} g_{\mu \nu}^{(n)}, n \leq 8$. (One would also expect that this gravitational interference or "Weyl field" of gravitational nonuniformity, corresponding to the interference in the double-slit set-up, would be exactly obscured from Alice's view by the Lorentz and gravitational shifts in her frame.) However, due to $h_{5} \lll r_{4}$, the 4-surface feels flat (i.e., Bob never experiences his own mass), and the apparent area singularity $\left(\ell_{P}^{2}\right)$ towards which he is falling therefore never leaves the $S^{3}$ horizon.

\subsection{Scheme for $Q G$ and the Information Paradox}

As Bob reaches the singularity in his frame in finite time, had his clock been intact, it would have recorded exactly $t_{(r=0)}\left(F_{B}\right)=t_{(r=2 G M)}\left(F_{A}\right)$. Thus the "central singularity” and "event horizon" are both horizons and are identified; $(\mathrm{A} \equiv \mathrm{B})$; i.e., the apparent separation of points $\mathrm{A}$ and $\mathrm{B}$ becomes more real and is the physical basis

${ }^{4}$ In Hamilton's river model [18], spacetime is in effect pouring over the edge of spacetime at $r=2 G M$. 
for entanglement. One would then expect that the IR cut-off in Alice's frame is directly proportional to the UV cut-off as Bob hits the singularity at effective perspectival convergence in his frame (which we think of as $r=0$ ). Therefore Hawking radiation corresponds to the collapse vector $\mathbf{R}$, so $\mathrm{HR}=\mathbf{R}$, and $r=2 G M$ is just $r=0$ "writ large", thus obviating the need for tunnelling or cosmological protection, since all "central singularities" must be "exposed" in this way.

At $\mathrm{D} 4 \mid \mathrm{D} 5$, the $\mathrm{D}(n-3)$ reduction is from $h_{5}$ to an apparent area $\left(\ell_{P}^{2}\right)$ singularity (at the macroscopic level, a black hole microstate, and at the microscopic level, a Dirac electron spinor). At D7|D8, the $\mathrm{D}(n-3)$ reduction is from $h_{8}$ to an apparent $\left(\ell_{P}^{5}\right)$ singularity (at the macroscopic level, a black hole, and at the microscopic level, an electron). Therefore, inter-electron spacing (charge quantisation) is defined in terms of photons, and inter-black hole spacing (gravitational quantisation) is defined in terms of gravitons. To non-gravitationally accelerate is to gain extra 5-height and thereby to force the D2 $\rightarrow$ D3 transition and reveal the D4|D5 embedding (a photon). Likewise, to measure is to gain extra 8-height and thereby to force the D5 $\rightarrow$ D6 transition, revealing the D7|D8 embedding (a graviton). (The act of measurement is therefore not one of "amplification" from the microscopic, quantum frame up to the macroscopic, classical frame, but one of projection in the opposite direction.) Thus to measure a black hole is effectively to overlay the transition D5 $\rightarrow$ D6 onto D2 $\rightarrow$ D3. Therefore, for Alice to measure any property of a black hole is to generate a shockwave [17] in the ER bridge between black hole throats in the maximally extended Schwarzchild solution, or to reveal the identification of the apparent D5 singularity $\ell_{P}^{5}$ (point A) with the actual D6 singularity $\ell_{P}^{6}$ (point B) and thereby produce a graviton (manifesting as a firewall) in the opposite throat.

For interference to occur in the double-slit set-up, the separate eigenstates representing the outcomes in which the particle passed through $\mathrm{SLIT}_{\mathrm{A}}$ or $\mathrm{SLIT}_{\mathrm{B}}$ are quantum linearly superposed; i.e., the wavefunction describes both states, $|\psi\rangle_{(A, B)}=1 / \sqrt{2}\left(|\psi\rangle_{(A)}+\mathrm{e}^{\mathrm{i} \theta}|\psi\rangle_{(B)}\right)$, where $\theta$ is the phase difference between the two paths. In the perspective model, a body's mass is the projection of its local 5-height to the remote frame, where it appears as the body's highly contracted (Schwarzchild) radius, $r_{S c h \text {. }}$, so $m_{B H}=\ell\left(S_{(A)}^{3}-S_{(B)}^{3}\right)$. Since points A $(r=0)$ and $\mathrm{B}(r=2 G M)$ are entangled horizons (i.e., identified), there is a spacetime path-length difference (phase) between frames $F_{A}$ and $F_{B}$ (so $m \propto \mathrm{e}^{\mathrm{i} \theta}$ ). By measuring the black hole, Alice is in effect deriving which-path information, destroying Weyl interference (i.e., the loss of the $2 \cos \theta$ term in Figure 2(b)) and thereby quantising the gravitational field. It was Einstein's happiest thought [20]-[22] that gravity is eliminated in a freely falling frame, and whilst classically, tidal forces cannot be gauged away, quantum gravitationally they are eliminated. In Alice's frame, Bob enters the graviton frame at $r=2 G M$, and, since no time elapses in the graviton frame, is instantly transported to $r=0$, and in his own frame, due to the temporal phase, he reaches $r=0$ and is instantly transported to $r=2 G M$ (i.e., in the conformally invariant limit, mass and time have no meaning [7]). At D2|D3, an object that disappears over the $S^{1}$ horizon does not cease to exist but is conserved in 3-space. Likewise, "falling into a black hole” is just a $\mathrm{D}(n-3)$ reduction, or projection from the local, macroscopic frame (Bob) to the remote, effectively microscopic frame $\left(\ell_{P}^{2}\right)$. Therefore, the information content of infalling matter defined in terms of inter-electron connectivity (i.e., photons) becomes inter-black hole connectivity (i.e., gravitons). In other words, information is preserved in $\mathbb{R}^{5}$ (or more correctly, $\mathbb{R}^{8}$ ), as gravitons exchanged by black holes "in black hole space" (in our D4 frame we might say "inside black holes", e.g., see [23]-[26]).

\subsection{The Origin of Black Hole Entropy}

The limit principle implies a "maximum physical reality" at D6, that D7 is therefore non-physical (i.e., mathematical) and that D8 is in some appropriate sense "beyond" mathematics (i.e., noncomputable). In the perspective model, Alice's highest freedom (i.e., her measurement independence, which by definition must be noncomputable [27]) is effectively projected into the remote frame where it appears as quantum indeterminacy. At D4 D5, local 5-height projected into the remote frame appears at the microscopic level as the coupling constant $2^{-1 / 2} M$ linking the Dirac spinors of the zigzag electron [5], and at the macroscopic level as the Schwarzchild radius $r_{\text {Sch. }}$ linking what in the conventional picture are $r=0$ and $r=2 G M$. At D7|D8, local 8- 
height projected into the remote frame appears at the microscopic level as EPR entanglement (the identification $\mathrm{A} \equiv \mathrm{B}$ ), and at the macroscopic level as an ER bridge. At D2|D3, an object that disappears over $S^{1}$ and returns from the opposite 2-space direction demonstrates spherical closure of the 2-surface. Likewise, at D4|D5, to accelerate is to gain extra 5-height, to see closure of D4 and, by Newton's third law, to invoke an equal and opposite reaction in which the by-product is heat (the fall-off of energy usefulness). At D7|D8, to measure is to gain extra 8-height, to see closure of D7 [28] and, by a quantum third law, to invoke an "equal and opposite reaction" (e.g., spin measured along the z-axes of spacelike separated EPR-entangled particles), in which the byproduct is entropy (the fall-off of information usefulness). In other words, "physical reality" must demonstrate at least the same degree of freedom in its response to measurement, as proposed in [29].

In the perspective model, a black hole is just the difference between spacetime path lengths; i.e., the effective separation of points A and B (conventionally, $r=0$ and $r=2 G M$ ), which is just $r_{\text {sch. }}$. For the freely falling asymptotic Alice, the apparent convergence limit $\ell_{P}^{2}$ represents a Hawking photon, or unit of projection. (Since freefall presumes minimum 5-height, the "emission rate" is also minimal; $(\mathrm{d} / \mathrm{d} t) \mathrm{HR} \propto \lambda$.)The infalling Bob encounters the perspectival convergence limit $\ell_{P}^{2}$ in his frame as a graviton (a firewall, or surface of maximum acceleration) at so-called $r=0$. Therefore, $N\left(\ell_{P}^{2}\right)=N\left(\gamma_{\mathrm{HR}}\right)$ is a measure of how many D4|D5 projection-units (Hawking photons) it takes (via $E=m c^{2}$ ) to disperse the black hole's mass, and $N\left(\ell_{P}^{2}\right)=N\left(\gamma_{\mathrm{G}}\right)$ is a measure of how many D7|D8 projection-units (inter-black hole gravitons) it takes to disperse the black hole's information. However, by projection or by infall (i.e., by $\mathrm{D}(n-3)$ reduction), a black hole is both $\boldsymbol{a}$ macroscopic and a microscopic entity, and therefore the path length difference (i.e., the separation of $r=0$ and $r=2 G M$ ) isa maximum of one graviton; $\ell[(r=2 G M)-(r=0)]=\gamma_{G}$. Thus a single graviton or unit of spacetime uncertainty accounts for the entire $r_{\text {Sch. }}$ distance (measured as noncomputability), which is of course proportional to entropy, expressed as $S_{B H}=c^{3} A / 4 G \hbar=k_{B} A / 4 \ell_{P}^{2}$ and calculated at $A=4 \pi r^{2}$ [11] [30]. This many-to-one reduction is an instance of the fact that quantum measurement reduces multiple complex-weighted, linearly superposed alternatives to a single classical outcome (in accordance with the Born rule, $p_{C}=|a|^{2}$ ).

Bob (or what remains of his body) always reaches the so-called $r=0$ (and is transported to the so-called $r=2 G M$, due to $\mathrm{A} \equiv \mathrm{B})^{5}$. Since he experiences no drama, the EP is not violated. Noting the similarity of the EP and a black hole (roughly, that neither reveals composition), it seems reasonable to claim that, since the EP is never violated, Hawking radiation cannot contain quantum information (i.e., is completely thermal).Information "loss" is then seen as an SM phase transition. Just as SU(3) colour bleaches out of Quarkworld (to be conserved in the form of colour-neutral hadrons), so electric charge $(+,-)$ bleaches out of D4 (to be conserved in the form of charge-neutral atoms defined in terms of photon-exchange between electrons), and "mass charge" (CON, EXP) bleaches out of D4 (to be conserved in the form of mass-charge-neutral "cosmic atoms" defined in terms of graviton-exchange between black holes).

\section{Discussion and Summary}

One would like to explore a number of further implications of the perspective model, including;

1) that the "geometry of time" (i.e., a potential $h_{n+1}$ above a spherically closed spacetime surface $\mathcal{M}^{n}$ ) is common to GR (classical spacetime at D4|D5) and QM (quantum spacetime at D7|D8),

2) that apparent flatness and collapse correspond to the $\mathbf{U}$ and $\mathbf{R}$ processes of $\mathrm{QM}$,

3) that at D7 $\mid \mathrm{D} 8$, assuming $h_{8} \lll r_{7}$, quantum superposed alternatives are multiple $|\psi\rangle$-collapse thresholds ( $S^{6}$ horizons) at one-graviton spacings, and that Schrödinger cat states collapse when associated spacetime curvature reaches a threshold of $\sim 1$ graviton $\approx m_{P} \approx 10^{-5}$ grams [26].

4) that if black holes correspond to paths, impacts on the second screen of the double-slit set-up should replicate the distribution of black holes in that direction,

\footnotetext{
${ }^{5}$ The graviton as "unit of spacetime uncertainty" accounts for what would otherwise be a causality-violating, backwards-in-time jump.
} 


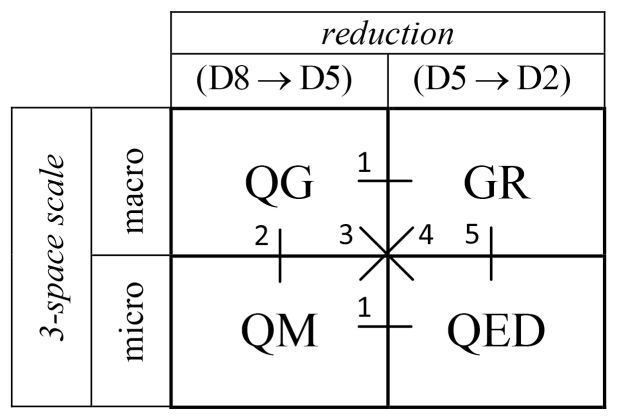

Figure 6. The four sectors defined by $\mathrm{D}(n-3)$ reductions and 3-space scale. Correspondences; (1) Born reciprocity, (2) ER $\leftrightarrow$ EPR duality, (3) ER $\leftrightarrow$ BCS duality, (4) CP ( $c \leftrightarrow \hbar$ duality) and (5) $B H \leftrightarrow e^{-}$duality.

5) that Newton's third law may be applied to EPR entanglement, in that the outcome of a measurement of spin along the $z$-axis of one entangled particle is perfectly correlated by the opposite outcome of a measurement of spin along the $z$-axis of the spacelike separated particle,

6) that, as at D2 $\mid \mathrm{D} 3$, apparent 3-height is maximum in the local frame and projected to an apparent minimum in the remote frame, so at D4|D5, the local "rate at which time passes" (i.e., maximised proper time, $\tau$ ) is projected to the remote frame and juxtaposed with "cosmic" time,

7) that the projection of local (macroscopic) values into the remote (effectively microscopic) frame explains apparent fine-tuning, naturalness, hierarchy and large number problems.

To summarise, the two principle $\mathrm{D}(n-3)$ reductions ( $\mathrm{D} 8 \rightarrow \mathrm{D} 5$ and $\mathrm{D} 5 \rightarrow \mathrm{D} 2$ ) and the two scale extremes (i.e., macroscopic and microscopic) define four sectors (Figure 6).

\section{Acknowledgements}

I am extremely grateful to Arkady Bolotin (Ben Gurion University of the Negev) for his comments, advice and patience.

\section{References}

[1] Lemaître, G. (1927) Annales de la Société Scientifique de Bruxelles (in French), 47, 49.

[2] Hubble, E. (1929) Proceedings of the National Academy of Sciences, 15, 168-173. http://dx.doi.org/10.1073/pnas.15.3.168

[3] Perlmutter, S., et al. (The Supernova Cosmology Project) (1999) The Astrophysical Journal, 517, 565-586. http://dx.doi.org/10.1086/307221

[4] Adam G. Reiss, et al. (Supernova Search Team) (1998) The Astrophysical Journal, 116, 1009-1038. http://dx.doi.org/10.1086/300499

[5] Penrose, R. (2004) The Road to Reality: A Complete Guide to the Laws of the Universe. Jonathan Cape, London.

[6] Penrose, R. (2006) Before the Big Bang: An Outrageous New Perspective and its Implications for Particle Physics. Edinburgh, Scotland: Proceedings of EPAC 2006, 2759-2767.

[7] Penrose, R. (2008) Causality, Quantum Theory and Cosmology. In: Majid, S., Ed., On Space and Time, Cambridge University Press, Cambridge, 141-195. http://dx.doi.org/10.1017/CBO9781139644259.004

[8] Burinskii, A. (2012) Kerr-Schild Geometry from Cosmology to Microworld and Space-Time Structure. arXiv:1212.5595v1 [physics.gen-ph]

[9] Burinskii, A. (2012) What Tells Gravity on the Shape and Size of an Electron. arXiv:1212.2920v1 [physics.gen-ph]

[10] Burinskii, A. (2012). Gravity vs. Quantum theory: Is Electron Really Pointlike? arXiv:1112.0225v2 [hep-th]

[11] Hawking, S.W. (1971) Physical Review Letters, 26, 1344-1346. http://dx.doi.org/10.1103/PhysRevLett.26.1344

[12] Susskind, L., Thorlacius, L. and Uglum, J. (1993) Physical Review D, 48, 3743. http://dx.doi.org/10.1103/PhysRevD.48.3743

[13] Stephens, C.R., 't Hooft, G. and Whiting, B.F. (1994) Classical and Quantum Gravity, 11, 621. 
http://dx.doi.org/10.1088/0264-9381/11/3/014

[14] Almheiri, A., Marolf, D., Polchinski, J. and Sully, J. (2013) Black Holes: Complementarity or Firewalls? arXiv:1207.3123v4[hep-th], [gr-qc]

[15] Maldacena, J. and Susskind, L. (2013) Cool Horizons for Entangled Black Holes. arXiv:1306.0533v2 [hep-th]

[16] Susskind, L. (2014). Computational Complexity and Black Hole Horizons. arXiv:1402.5674v2 [gr-qc], [quant-ph]

[17] Stanford, D. and Susskind, L. (2014) Complexity and Shock Wave Geometries. arXiv:1406.2678v2 [hep-th]

[18] Hamilton, J.S. and Lisle, J.P. (2004) The River Model of Black Holes. arXiv:gr-qc/0411060v2

[19] Hamilton, J.S. (2012) Illusory Horizons, Thermodynamics, and Holography inside Black Hole. arXiv:1210.4541v1 [gr-qc]

[20] Einstein, A. (1907) Jahrbuch der Radioaktivität (Yearbook of Radioactivity), 4, 411-462 (Wir betrachen zwei Bewegung Systeme).

[21] Schucking, E. (2012) Einstein’s Apple: His First Principle of Equivalence. arXiv:gr-qc/0703149v2

[22] Pais, A. (1982) Subtle Is the Lord: The Science and the Life of Albert Einstein. Oxford University Press, Oxford, 178.

[23] Marolf, D. (2009) Black Holes, AdS, and CFTs. arXiv:0810.4886v2 [gr-qc]

[24] Hsu, S.D.H. and Reeb, D. (2009) Monsters, Black Holes and the Statistical Mechanics of Gravity. arXiv:0908.1265v1 [gr-qc]

[25] Christodoulou, M. and Rovelli, C. (2014) How Big Is a Black Hole? arXiv:1411.2854 [gr-qc]

[26] Ong, Y.C. (2015) Never Judge a Black Hole by Its Area. arXiv:1503.01092v4 [gr-qc]

[27] Penrose, R. (1989) The Emperor's New Mind: Concerning Computers, Minds and the Laws of Physics. Oxford University Press, Oxford.

[28] Gödel, K. (1931) Über Formal Unentscheidbare Sätze der Principia Mathematica und Verwandter Systeme, I. and On Formally Undecidable Propositions of Principia Mathematica and Related Systems I in Solomon Feferman, ed. (1986) Kurt Gödel Collected Works, Vol. I. Oxford University Press, Oxford, 144-195.

[29] Conway, J. and Kochen, S. (2006) Foundations of Physic, 36, 1441. http://dx.doi.org/10.1007/s10701-006-9068-6

[30] Bekenstein, J.D. (1973) Physical Review Letters, D7, 2333. http://dx.doi.org/10.1103/PhysRevD.7.2333 